Visión Antataura

Vol.4, No.2: 153-168

Diciembre 2020 - Mayo 2021

Panamá

ISSN 2520-9892

\title{
Sobre el turismo en Panamá después del COVID-19: el rol de la prospectiva en las políticas públicas
}

\section{On Tourism after the Covid-19 in Panama: the role of foresight in public policies}

\author{
Nelly del Carmen Araya Reyes ${ }^{1}$
}

${ }^{1}$ Doctorado en Gestión de la Hospitalidad con Énfasis en Administración de Hoteles y Turismo; Profesora, Universidad de Panamá, Facultad de Administración de Empresas y Contabilidad, Departamento de Administración de Empresas Turísticas; nellydelc.araya@up.ac.pa; https://orcid.org/0000-0001-9369-0239

Resumen: La incertidumbre sobre lo que pueda regir en el futuro de la actividad turística después del COVID19 ha traído un debate académico y profesional en los últimos meses en Panamá y el mundo, esta imposibilidad de prever lo que pueda ocurrir para el país tiene un elevado costo en cuanto a la capacidad de diseñar las políticas correctas, o al menos más viables, para superar la situación actual del sector, que en este momento se encuentra sumido en una gran caída de su valor económico y social producto de la pandemia. Asimismo, representa un panorama distinto, el de no estar preparado para otro evento que pudiera ser aprovechable o debiera ser mitigable. En este contexto, los estudios de prospectiva han probado ser eficientes en la integración de esfuerzos para esbozar esos posibles futuros, sobre bases metodológicas y de conocimiento. Esta investigación, de tipo documental, procura evidenciar la necesidad que tiene Panamá de aplicar esta herramienta en el turismo post COVID-19 a fin de contar con mayores y mejores bases para la toma de decisiones. La investigación corroboró la necesidad de crear escenarios turísticos posibles, probables o improbables, para la formulación de políticas públicas en el sector y la utilidad de la prospectiva para ello.

Palabras clave: turismo post COVID-19, políticas públicas, prospectiva

\begin{abstract}
The uncertainty about what may govern the future of tourism activity after COVID -19, has brought an academic and professional debate in recent months in Panama and the world, this impossibility of foreseeing what may happen, for the country has a high cost in terms of the ability to design the right policies, or at least more viable, to overcome the current situation of the sector, which is currently immersed in a great fall in its economic and social value due to the pandemic. Likewise, it represents a great risk; that of not being prepared for another event that could be exploited or should be mitigated. In this context, foresight studies have proven to be efficient in integrating efforts to outline these possible futures, on methodological and knowledge bases; this research, of a documentary nature, seeks to highlight the need for Panama to apply this tool in post-COVID-19 tourism, in order to have greater and better bases for decision making. The research corroborated the need to create possible, probable or improbable tourism scenarios for the formulation of public policies in the sector and the usefulness of foresight for this purpose.
\end{abstract}

Keywords: post COVID-19 tourism- 19, public policies, foresight. 


\section{Introducción}

No cabe la menor duda de que el sistema de turismo es uno de los más vulnerables a las crisis, puesto que las actividades turísticas se encuentran interrelacionadas y dependen de sectores como el transporte, la climatología, el cambio de moneda, las políticas públicas, los órdenes sociales del país de partida y el de llegada, etc. Referentes como el brote de fiebre aftosa del Reino Unido en el año 2000, o el síndrome respiratorio agudo grave (SARS) en 2000 han sido la evidencia más clara de las consecuencias para esta importante industria.

Aventurarse a entender el panorama del turismo después de la cuarentena a causa del COVID-19 es atisbar sus efectos a largo plazo, desde el punto de vista académico, profesional y empresarial, se plantean las interrogantes: ¿Cómo se comportará el turista? ¿Cómo recuperar mercados? ¿Valdrá la pena reinventar un sistema que se encuentra en pausa obligatoria extremando recursos financieros y humanos?, ¿Se deberá poner en movimiento el aparataje turístico después de una crisis jamás imaginada? ¿Aprender a operar con la amenaza constante del virus?

El turismo es, sin duda alguna, una actividad que ha reflejado históricamente un crecimiento sostenido, en las figuras 1 y 2 lo demuestra la Organización Mundial del Turismo (OMT) como parte de su reporte Panorama del Turismo Internacional, Edición 2019 (UNWTO, 2019).

Figura 1. Llegada de turistas internacionales e ingresos por turismo internacional (porcentaje de variación)

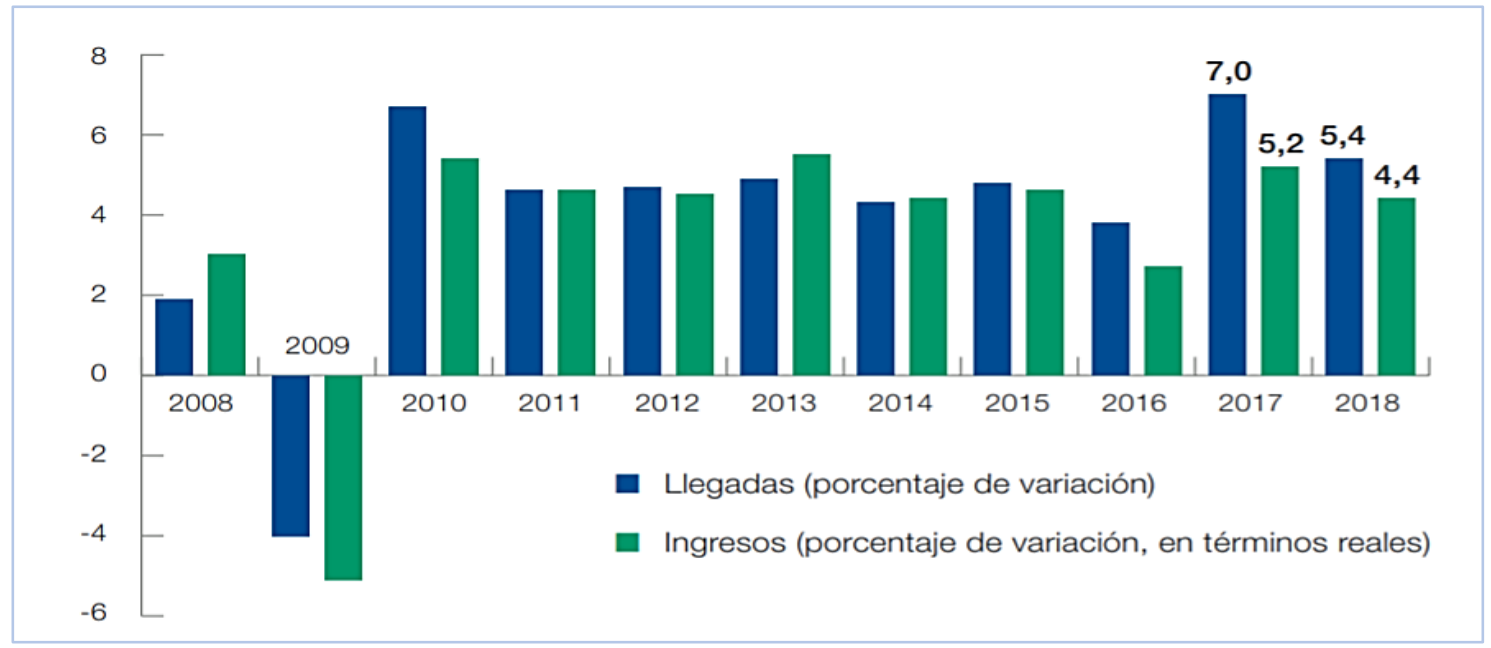

Fuente: Organización Mundial del Turismo (OMT-UNWTO), julio de 2019 
Figura 2. Llegada de turistas internacionales e ingresos por turismo internacional (porcentaje de variación)

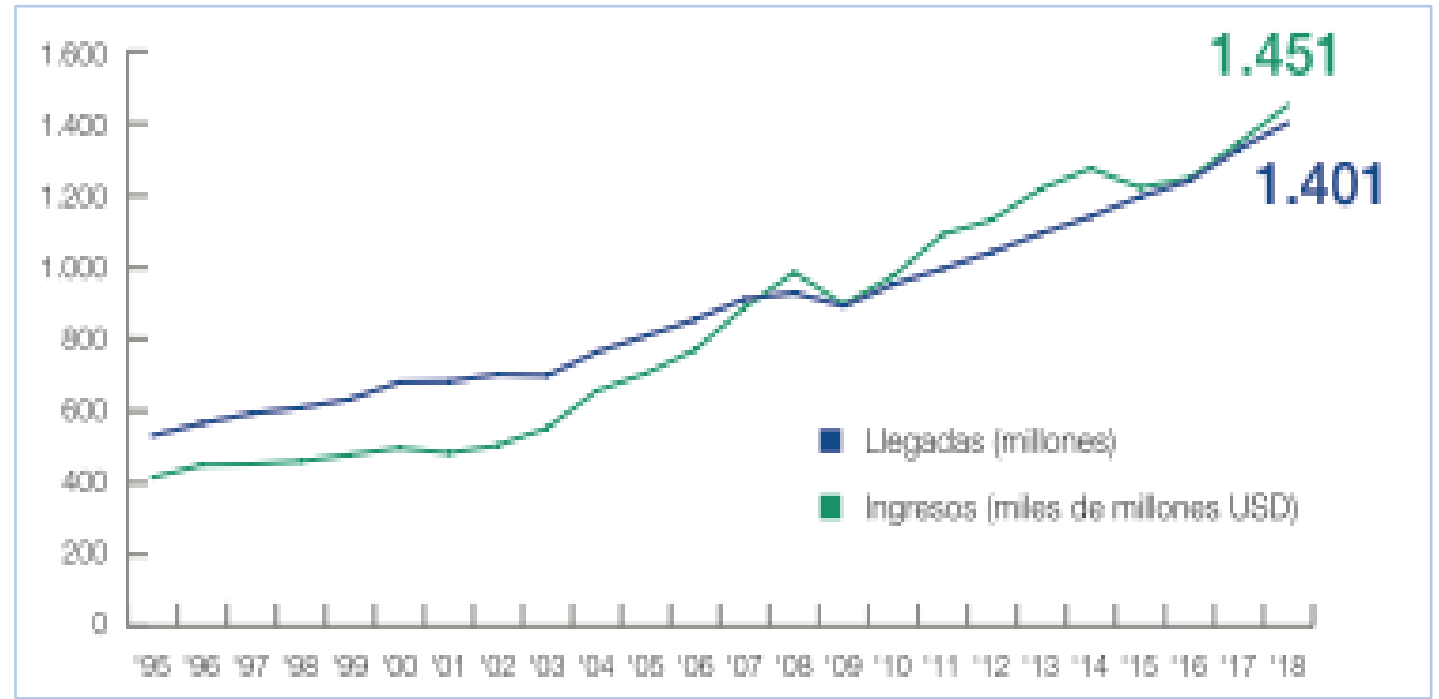

Fuente: OMT, 2019.

En el año 2018, la actividad turística se encontraba en un contexto que propiciaba el aumento de su valor, tanto en términos financieros, como en empleo y otras externalidades positivas en el ámbito social; existía un ambiente económico favorable, con un Producto Interno Bruto (PIB) mundial superior al 3,6\% y con tipos de cambio que presentaban variaciones moderadas e intereses bajos. Por otro lado, el momento se caracterizaba por una fuerte demanda de vuelos, un aumento del 6\% en tráfico de pasajeros internacionales, con precios estables en combustibles y tarifas 60\% más bajas en relación al año 1998 (UNWTO, 2019).

Sin embargo, a pesar del crecimiento continuo que se evidencia desde 1995 y que se muestra en las figuras 1 y 2 , hoy, el sector está siendo sometido a un stress pocas veces visto, relacionado al impacto de la pandemia por COVID-19, en torno a ello las Naciones Unidas en su página COVID-19. Respuesta (ONU, 2020) declaran que:

EI COVID-19 ha alterado la vida de miles de millones de personas y ha puesto en peligro la economía mundial. El Fondo Monetario Internacional (FMI) ha anunciado una recesión global y la Organización Internacional del Trabajo (OIT) pronostica que para el segundo trimestre de 2020 se habrán perdido a nivel mundial un número de horas de trabajo equivalentes a las de 195 millones de trabajadores a tiempo completo. Y para finales de año, se habrán perdido salarios por valor de 3,4 billones 
de dólares. Los confinamientos, totales o parciales, afectan a casi 2700 millones de trabajadores, lo que representa alrededor del $81 \%$ de la población activa mundial (p.1).

En América Latina y el Caribe, el impacto del COVID-19 es particularmente intenso, y así lo demuestra la Comisión Económica para América Latina y el Caribe (CEPAL), que aclara que la región recibe la pandemia con el menor crecimiento económico de las últimas décadas y poco espacio de acción en política fiscal, afirmando que esta ocasionará la peor contracción desde 1900 para la región (CEPAL, 2020).

Volviendo al sector turístico, se espera una contracción de alrededor del 22\% en el primer trimestre y una caída de 60-80\% en el año para el turismo internacional (UNWTO, 2020). Con esto se puede observar que, con una disminución del $30 \%$ este año de los ingresos turísticos, pudiera significar una reducción del PIB de 2,5, 0,8 y 0,3 puntos porcentuales en el Caribe, México, Centroamérica y América del Sur, respectivamente y esto traería mayores repercusiones en el empleo, ingresos familiares y gubernamentales en el Caribe, especialmente en las micro y pequeñas empresas, que representan el 99\% de las empresas y el 77\% del empleo del sector de hoteles y restaurantes (CEPAL, 2020).

Por todo ello, urge tomar medidas que, a la par de propiciar la recuperación del sector turístico y de hospitalidad en Panamá, también reduzcan la posibilidad de que futuros eventos, puedan afectar nuevamente la actividad en forma tan dramática.

Esta investigación, revisó una de estas medidas en el ámbito de las políticas públicas para Panamá: valiéndose del uso de la prospectiva, entendida como “...la elaboración de métodos, procesos y sistemas prospectivos que permiten formular interrogaciones sistemáticas y organizadas acerca de los futuros posibles de la sociedad" como lo dejan ver Máttar y Perrotti, 2014, p. 176.

En ese sentido, se plantearon las siguientes interrogantes:

¿Cómo el sector privado y público logrará incentivar al turista en busca de la reactivación de este sistema de importancia notable en Panamá?

¿Cuál es el papel de la prospectiva en las políticas públicas y el Plan maestro de turismo sostenible al 2025 de Panamá para la reactivación del turismo post COVID- 19? 
Para lo cual este estudio se propuso como objetivo general:

Revisar documentalmente la importancia de los estudios prospectivos que sustentan la elaboración de políticas públicas para la reactivación del turismo post COVID-19 en Panamá.

\section{Abordaje metodológico}

Esta investigación es de tipo documental o bibliográfica, igualmente se considera exploratoria, y transeccional, ya que se ejecutó con un corte o línea en el tiempo para el año 2020 con respecto a la documentación revisada.

En principio se efectuó una revisión preliminar entre las principales bases académicas, se eligieron los documentos más relevantes, que aportaron información sobre el tema y los tópicos de la investigación y se priorizaron documentos de organizaciones como la OMT, OEA, CEPAL, ATP y CAMTUR además de artículos de prensa que brindaron noticias actuales en la temática.

Por último, se revisaron los documentos hasta que se consideró haber obtenido la información necesaria para responder a las interrogantes de investigación.

\section{Referentes teóricos}

\subsection{Sobre la formulación y análisis de políticas públicas}

Cómo el sector privado y público logrará incentivar al turista en busca de la reactivación de este sistema de importancia notable en Panamá.

Toda vez que aún no se cuenta con una vacuna contra el COVID-19, se optaría por aperturar gradualmente los sectores inherentes al turismo, puesto que se vislumbra un panorama poco alentador donde los principales afectados son los miles de empleos directos e indirectos que de ello dependen.

Desde la perspectiva de la administración, la política pública puede entenderse en el marco de la planificación como: donde "lo público" es aquello que involucra a toda la sociedad, y que encuentra su punto común en la estructura gubernamental, pudiera definirse entonces como planes o cursos de acción, generales y a largo plazo, implementados por el estado para alcanzar objetivos relacionados con el interés público. 
En todo caso, esos objetivos propuestos deben tener alcance social; es decir, abarcar los intereses del colectivo de una nación, no obstante, en la realidad, las políticas públicas están fuertemente comprometidas por intereses de clase, sectores o grupos de presión, los cuales no siempre representan las necesidades o requerimientos de la mayoría.

En principio, las políticas públicas deberían analizarse a través de su capacidad y utilidad para resolver problemas de la sociedad. Un análisis objetivo de ellas debería estudiar no solamente lo retórico, lo discursivo, sino considerar los propósitos reales, no siempre declarados, de los actores detrás de estas.

En síntesis, el análisis de políticas públicas, siendo este un proceso intelectual, inmerso en la actividad política, está determinado por condiciones objetivas de la sociedad, es decir situaciones problemáticas que deben ser resueltas o mejoradas (vialidad, educación, contaminación, resguardo de atractivos...) y que se combinan con los intereses políticos, visión, filosofía, etc., de los actores a la hora de caracterizar dicha situación (condiciones subjetivas); entonces, un problema llama la atención de los hacedores de política, cuando, de acuerdo con ambos tipos de condiciones, este debe ser resuelto.

Afirma Dunn (2012) que el análisis de políticas desde esta perspectiva, no se ocupa del conocimiento per se sino que ataca lo que los diferentes actores sociales consideran una oportunidad para mejorar o mantener, para la sociedad su calidad de vida o bienestar social, y para los responsables del análisis, su ejercicio de poder político y autoridad legal.

En Panamá, el turismo tiene el estandarte que lo resalta como una de las bases de su sostenimiento económico, del cual nacen diversas actividades relacionadas entre sí, esto ha permitido que la distribución de la riqueza sea pensada en pro del desarrollo de la estabilidad económica local, por medio de fuentes de trabajo, haciendo de este un país competitivo y productivo.

En el año 2017, se desarrolla el primer Plan Maestro de Turismo Sostenible (20072020) cuyo fin principal es poner valor a la oferta diferenciadora del país. Sin embargo, se desarrolló una actualización de dicho plan (2020-2025), dentro del cual se incluye lo siguiente: 
Durante el proceso de preparación de esta actualización ha tenido lugar la peor crisis del sector turístico mundial en la historia, a raíz de la pandemia global por corona virus (el llamado COVID-19). El parón en la actividad, de duración incierta a la redacción de estos textos, ha obligado a revisar los objetivos, las estrategias, y las acciones, adaptándolas a un mercado muy diferente del que había cuando se iniciaron los trabajos de actualización del Plan Maestro (p.7).

Es, debido a esto que se debe ajustar ciertas prospecciones realizadas en el Plan Maestro que van desde la infraestructura y gestión urbana (agua, alcantarillado, residuos sólidos, movilidad, espacio público y medidas de adaptación al cambio climático, así como el desarrollo de planes de desarrollo territorial, y el monitoreo de la movilidad y seguridad a través del uso de nuevas tecnologías, todo en función de la nueva normalidad por la pandemia del COVID-19. En tanto que el equipamiento, gestión turística local y desarrollo comunitario, que incluye muelles, centros de interpretación, y sistemas de señalización debe fortalecerse en base a la realidad de distanciamiento social emanada de la Organización Mundial de la Salud.

Otro de los cambios que deberá realizarse es en cuanto a materia de coordinación interinstitucional, que incluye el monitoreo ambiental el cual deberá enfocarse en la detección de posibles virus, y nuevas enfermedades zoonóticas o no, para apurar su prevención incluyendo el monitoreo ambiental.

Buena parte de las preferencias de los turistas se enfocan en las áreas protegidas del país, sean estos marinos o terrestres, esto nos da una idea de la importancia del recurso natural panameño y su relevante interrelación con la actividad turística, en el tiempo postCovid-19 actividades como el turismo náutico, pesca deportiva, juegos de azar, turismo cultural, visitas a pozos termales, turismo médico o wellness, visitas a comunidades indígenas, etc. se deberán realizar bajo un marco de cuidados extremos, tomando en cuenta el aforo correspondiente.

El marco post-COVID-19 hace necesario un esfuerzo suplementario en destinos y empresas turísticas para garantizar unas condiciones de seguridad (sanitaria) extraordinarias, muchas de las cuales requerirán modificaciones en las infraestructuras o el 
modus operandi, esto, supondrá, que los micro empresarios y emprendedores acudan a canales de crédito para solventar las inversiones, situación que muchas veces no es fácil, no se concreta debido a los variados requerimientos que solicitan las entidades financieras, trámite que se vuelve muchas veces engorroso y dilatado, por ello, las políticas públicas, deben incluir facilitar estos financiamientos.

El primer capítulo del libro TPC - 19 Turismo post COVID-19, de la Asociación Española de Expertos Científicos en Turismo (AECIT) está dedicado a la construcción de escenarios, entre algunos de ellos se encuentran "Reconfiguración del Sector", esto conlleva cambios de estructura en la acción de los gobiernos y en el comportamiento del turista.

En el caso de Panamá, el sector realizaría una reconfiguración de su oferta para adaptarse a estos cambios de índole cuantitativa y cualitativa, algo así como "reinventarse o desaparecer", para ello es fundamental, casi imprescindible que las empresas y destinos inspiren confianza y seguridad, aspectos que mitigarían el temor de viajar de los turistas, esto sumado a la actitud de los habitantes en los poblados receptores de visitantes. "Cambio cualitativo de la demanda turística", en este escenario el comportamiento del turista se viera afectado en la medida que las políticas estatales siguieran frenando los flujos de viajeros, también podría alimentar esta situación, favorecedora del turismo doméstico o de proximidad. En cualquier caso, sería una demanda inicialmente limitada y condicionada por la crisis económica derivada de la sanitaria. "Vuelta a la situación pre-COVID-19" con una política gubernamental de restricción de circulación, y un turista más cuidadoso en temas de salud y seguridad (Vargas Sánchez, 2020).

\subsection{Sobre la planificación y la prospectiva}

Bitar (2013) expresa que: si bien el futuro es impredecible, es necesario identificar posibles escenarios, a fin de suponer las consecuencias que eventos no considerados

pudieran acarrear, estos escenarios son estudiados por la prospectiva, para que la planificación, construya rutas en consecuencia. 
La prospectiva, como señala Mattar y Perroni (2014) se enfoca actualmente en la innovación, el cambio institucional y la adaptación al cambio por parte de las sociedades, esta procura:

- Optimizar la planificación en condiciones de sistemas complejos;

- Apoyar en los procesos de preparación, formulación de políticas y

- Ofrecer información y conocimiento para la planificación de largo plazo.

Originalmente, la prospectiva clásica se caracterizó por estar orientada al anticipo y reflexión sobre los "futuros posibles, probables y deseables". En la actualidad, además, se espera intervenir en tejidos institucionales y sociales, construyendo el devenir mediante decisiones estratégicas, la prospectiva comparte así con la planificación, el hecho de alinear la gestión gubernamental y la acción colectiva a tal fin, la concepción original de la prospectiva como anticipación (exploración de futuros posibles para la toma de decisiones en el presente), ha derivado en la construcción social del futuro, involucrando a todos los actores sociales (Máttar y Perrotti, 2014).

Panamá ha establecido sesiones del Grupo de Trabajo Virtual para buscar medidas y así apoyar el sector de turismo de crisis Covid-19. Las sesiones virtuales público-privadas buscan desarrollar medidas de apoyo y acciones para beneficiar al sector turístico durante y después de la crisis COVID-19 (OEA, 2020).

Estas mesas de trabajo virtuales enfocan distintas medidas de apoyo durante y después de la crisis por el COVID-19 su objetivo es canalizar los recursos necesarios para salvaguardar a la población en general, la salud de sus habitantes, generar bonificaciones dirigidas a las personas de bajos recursos para garantizar el sistema de alimentación; otro beneficio en el que se ha trabajado es el referente al manejo de la moratoria de las personas que adquirieron una o varias obligaciones económicas con bancos y otras empresas a priori de la pandemia. La banca panameña decidió otorgar tres meses de gracias para que sus clientes se pongan al día el pago de hipotecas, tarjetas de créditos, préstamos personales y comerciales, pago de cuotas de vehículos, maquinarias y herramientas de trabajo, etc. Esto se constituye en un colchón de soporte que da un aliciente a los emprendedores, empresarios y ciudadanía en general a salir adelante luego de una crisis sin precedentes que 
los ha obligado a vivir confinados. Resultaría efectivo así mismo prorrogar los incentivos fiscales a las empresas turísticas hasta el año 2025.

\section{3. ¿Cómo encuentra el COVID-19 a Panamá en materia de turismo?}

Para el año 2019, el Instituto Nacional de Estadística y Censo (INEC Panamá, 2019) refleja:

- Se recibieron 2.203.000 viajeros, de los cuales, 545.000 fueron turistas, 74.000 excursionistas, y 28.000 pasajeros de cruceros, para un total de gastos efectuados de 1.388.818.000 balboas.

- Un crecimiento total de $1.5 \%$ en relación con el año 2018 , con aumento del $8.3 \%$ en el ingreso de turistas, pero la caída del $13.7 \%$ de los excursionistas y el $51.8 \%$ de los pasajeros de cruceros, generando una variación positiva de 0.8 en los gastos efectuados. ¿Qué significa esto? En boca del ministro de turismo Iván Eskildsen: "Se han perdido 40.000 empleos en los últimos 20 meses. Es crítico. Hay hoteles que sufren atrasos en sus pagos bancarios. Hay mucha preocupación y mucha frustración" (EFE Panamá, 2019, p.1).

Igualmente, en la nota de prensa se reconoce que la ocupación en establecimientos de hotelería fue del $44,9 \%$ en 2018 , un 2,2 \% menos comparado con el año anterior, de acuerdo con la Autoridad de Turismo de Panamá (ATP), responsabilizando de la crisis al exiguo turismo doméstico y la falta de promoción en el mercado internacional, aun cuando en el 2017 fue creado un fondo público-privado para promover Panamá internacionalmente, habiendo recibido ya 5 millones de dólares para sus operaciones (EFE Panamá, 2019).

En materia de información y estadísticas, es necesario resaltar que el último avance de la Cuenta Satélite del Turismo de la República de Panamá (CSTP) publicada, es de los años 2007-2013, y está referida a gastos de visitantes, categoría de productos, consumo en el sector, cuentas de producción de la actividad, valores agregados, la oferta y el empleo turístico (INEC Panamá, 2019). Y en lo referente a planificación, lo vigente es el Plan Maestro de Turismo Sostenible de Panamá 2007-2020 (Instituto Panameño de Turismo, 2008). 


\section{4. ¿Cuál es el papel de la prospectiva en las políticas públicas y el Plan maestro de turismo sostenible al 2025 de Panamá para el Turismo post-COVID-19?}

En el taller de reflexión del estudio de prospectiva "Panamá 2040. Visión de un país exitoso" organizado por la Secretaría Nacional de Ciencia, Tecnología e Innovación (SENACYT) en el año 2018, se explora las tendencias futuras de Panamá dentro de 20 años, examina cuatro posibles escenarios del país que presentan una imagen alternativa de cómo podría evolucionar la nación, la ciencia, la tecnología y la innovación pretenden convertirse en una estrategia de país para conseguir una sociedad más inclusiva y sostenible, que los personajes claves trabajen en conjunto, compartiendo la misma visión, significa construir un escenario futuro lleno de alternativas visibles.

La Magister Candanedo, Jefa de la Oficina de Planificación de la SENACYT quien lidera el estudio, expuso que Panamá es una sociedad “...no basada en el conocimiento, sino en el mercado, y en 'drivers' que no necesariamente enfatizan el conocimiento", reiterando que La Política Nacional de Ciencia, Tecnología e Innovación y el Plan Nacional 2015-2019 (PENCYT) tiene años planteando la necesidad de fundamentarse en el conocimiento, (SENACYT, 2018).

Este estudio forma parte del proceso de preparación de escenarios con visión hacia el año 2040, utilizando la técnica de foresight el cual es un proceso sistemático de reflexión y construcción de una visión del futuro entre un grupo de actores del desarrollo, cuyo resultado debe servir como insumo para el diseño de la próxima Política Nacional de Ciencia, Tecnología e Innovación y Plan Nacional (PENCYT) 2020-2040.

El estudio prospectivo citado, presentó como principales hallazgos en cuanto a los posibles escenarios futuros:

1. Aumento del empleo informal aumentará al año 2040 y disminución del $32.8 \%$ de la diversificación productiva. El aporte del sector servicios al Producto Interno Bruto (PIB) se mantendrá igual, y aumento del PIB nacional por la contribución de este sector.

2. Aumento del $73.7 \%$ en el envejecimiento poblacional y disminución en el acceso a la seguridad social de los ciudadanos disminuirá. Mantenimiento del índice de progreso social para el 2040. 
3. Aumento del efecto de cambio climático al 2040 , disminución de la biodiversidad fuera de las áreas protegidas, y sostenimiento de la optimización del recurso hídrico del Canal de Panamá al 2040.

4. Aumento de los gastos de capital del sector público para el 2040, disminución de la independencia de los poderes del Estado con mantenimiento de la debilidad del sistema jurídico.

De acuerdo con el doctor Carlos Aguirre, Asesor de la Oficina de Planificación de la SENACYT y especialista en Políticas Públicas de Ciencia y Tecnología, los escenarios construidos para Panamá en 2040 en cada uno de los drivers son:

1. Escenario A "Cambio estructural con igualdad".

2. Escenario B "Vivir bien".

3. Escenario C "Más de lo mismo".

4. Escenario D "Peor que antes".

Se coincide con el punto de vista del mencionado asesor, donde los escenarios son descripciones posibles del futuro, sin embargo, no deben ser considerados pronósticos, ni predicciones, mucho menos planificaciones a largo plazo. El escenario es más bien una historia del futuro, y sobre esta se debe estimular un debate sobre las alternativas del país para reactivar sus principales, por no decir todos sus frentes económicos.

Compárese esto con las palabras de Iván Eskildsen, quien en la presentación del avance del Plan Maestro de Turismo Sostenible post COVID-19 declaró que este parte de la visión de “...un destino turístico sostenible de clase mundial, gracias a la extraordinaria riqueza y diversidad de nuestro patrimonio natural y cultural, así como la calidad de nuestros servicios" (Redacción Excelencias News Panamá, 2020).

En este momento de transición para el mundo y particularmente para Panamá, el papel de los actores involucrados en la reactivación turística es sumamente importante, los empresarios, trabajadores, gestores y turistas se verán llamados a replantearse el funcionamiento, la gestión, las estructuras empresariales y las propias relaciones del sector turístico, dentro de ese trabajo mancomunado, el uso de la las tecnologías será 
fundamental, dichos actores serian entonces, engranaje para el cambio de la matriz productiva enfocada en aspectos ambientales y sociales.

Siendo así, el marketing de destinos podría ser empleado como herramienta para posicionar a Panamá entre los principales destinos en Latinoamérica y de la región y entre los actores de la reactivación económica y turística. Propiciar los espacios de colaboración con grupos de interés es una de las opciones para conseguir el éxito del destino, para esto, es imperativo que prevalezca la autonomía de las organizaciones de mercadeo de destino DMO's, con el objetivo de que los proyectos planteados sean sostenibles a largo plazo, manejados por los mejores profesionales con trayectoria para liderar la organización, usando como ejemplo el caso de la ciudad de Toronto en Canadá, la cual recientemente incorporó como presidente y CEO de su DMO al estadounidense Scott Beck, quien ejerció el mismo rol durante 14 años en la ciudad de Salt Lake City, Utah, y en tan solo 90 días de su incorporación ya se perciben resultados.

Este es un momento clave para para cambiar la historia del turismo en Panamá, si se elige una adecuada estrategia de mercadeo de destino, esta impactará en la economía, porque mejoraría inicialmente la calidad de vida de la población, elevando el perfil del destino en cuanto a sus inversiones y convirtiéndose en un imán para llevar a cabo eventos estratégicos como convenciones, simposios y conferencias, actividades que ya de por si dejan ver el reflejo socioeconómico por la cantidad de visitantes y reservas en hoteles, o centros de hospedaje.

Nótese que la decisión sobre el tipo de destino turístico que se desea para Panamá es uno basado en el producto que se supone "o se quisiera" tener, en contradicción con lo proyectado por la SENACYT cuando se refiere a tomar decisiones sobre la base del "conocimiento", de ahí la importancia de construir escenarios a partir de estudios de prospectiva. Un ejemplo de ello: existen cuatro transformaciones profundas que no se previeron adecuadamente y que han afectado al continente y por ende a Panamá: el surgimiento del internet, el crecimiento de la influencia económica de China, la crisis financiera reciente y el cambio climático (Máttar y Cuervo, 2016), agréguesele ahora el 
COVID-19 y quedará claro por qué es necesario construir escenarios para apoyar la planificación estratégica del país en general y del sector turístico en particular.

En resumen, ante la incertidumbre, con el razonamiento que propone la prospectiva, se pueden seguir las tendencias mundiales principales y asimilar los acontecimientos con poca probabilidad, pero alto impacto de llegar a ocurrir.

\section{Conclusiones}

- La investigación demostró que es necesario para nuestro país, diseñar posibles escenarios sobre la base del conocimiento, que permitan prever situaciones de otra forma imposible de hacer, y que fortalezcan su capacidad de tomar decisiones y articular políticas públicas coherentes y adecuadas, concluyéndose en definitiva que los estudios de prospectiva, parecieran ser la herramienta más eficiente para tal efecto.

- Sin duda alguna existirá un antes y un después en el sector turístico panameño que dependerá netamente de la planificación estratégica empresarial y modelos de negocios que se pretendan propiciar.

- Para el caso panameño, ¿cómo saber que escenarios considerar? Aparece claramente entonces la conclusión sobre la necesidad de poder elaborar estos posibles escenarios para el país, de ahí que este artículo más que "predecir" como podrá ser el turismo post COVID-19, enfatiza en la necesidad de aplicar la prospectiva para contar con herramientas que permitan esbozar visiones de futuros posibles, con razonable estructuración y horizontes estratégicos, orientando un modelo propio de desarrollo turístico sostenible.

- Otra conclusión que se desprende de la investigación es la capacidad que ostenta la prospectiva para la elaboración y el diseño estratégico de las políticas públicas, con capacidad para brindar sentido y fortaleza a la dimensión político/institucional del turismo en Panamá.

- Finalmente, se puntualiza la utilidad de la prospectiva para la promoción del diálogo social permanente entre todos los actores, sector privado, público y sociedad, para modelar el futuro que necesita Panamá, sobre los hechos de su presente. 


\section{Referencias bibliográficas}

Bitar, S. (2013). Why and how Latin America should think about the future. Inter-American Dialogue, (September), 52.

CEPAL. (2020). Informe sobre el impacto económico en América Latina y el Caribe de la enfermedad por coronavirus (COVID-19): estudio elaborado por la Comisión Económica para América Latina y el Caribe (CEPAL) en respuesta a la solicitud realizada por el Gobierno de México. Santiago de Chile: Comisión Económica para América Latina y el Caribe (CEPAL).

Dunn, W. (2012). Public policy analysis (5th ed.). New Jersey: Pearson Education Inc.

EFE Panamá. (2019). El nuevo ministro de turismo de Panamá dice que la situación del sector es "crítica." Recuperado el 15 de junio de 2020 de El Diario.es: https://www.eldiario.es/economia/ministro-Turismo-Panama-situacion-

\section{critica 0 919008102.html}

INEC. Panamá. (2019). Instituto Nacional de Estadística y Censo. Recuperado de: https://www.inec.gob.pa/

Instituto Panameño de Turismo. (2008). Plan maestro de turismo sostenible de Panamá 2007-2020 - Instituto Panameño de Turismo. Recuperado de: https://www.atp.gob.pa/analisis-diagnostico-general-del-turismo-en-panama

Máttar, J., y Cuervo, M. (2016). Por qué América Latina y el Caribe debe pensar en el futuro. In Planificación y prospectiva para la construcción de futuro en América Latina y el Caribe (p. 221). Santiago de Chile: Naciones Unidas.

Máttar, J., y Perrotti, D. E. (2014). Planificación, prospectiva y gestión pública: Reflexiones para la agenda de desarrollo. Recuperado de:: https://repositorio.cepal.org/bitstream/handle/11362/36762/1/S20131070 es.pdf

OEA. (2020). Boletín de Cultura y Turismo: COVID-19 respuestas de política turística (A partir del 7 de abril de 2020) Recuperado de: https://www.tvn2.com/nacionales/Coronavirus-en-Panama-Mesas-trabajo-virtuales-medidasCOVID19-Ivan-Eskildsen-ATP-turismo 0 5543445623.html

ONU. (2020). La ONU pide medidas para amortiguar el impacto del COVID-19 en el mercado 
168. Visión Antataura, Vol.4, No.2, Diciembre 2020 - Mayo 2021

$\begin{array}{lllll}\text { laboral. } & \text { Recuperado } & \text { de: }\end{array}$ https://www.un.org/es/coronavirus/articles/impacto-covid-19-mercado-laboral

Redacción Excelencias News Panamá. (2020). Panamá: presentan plan maestro de turismo sostenible post COVID-19. Recuperado de: Excelencias News Panamá: https://www.excelenciaspanama.com/turismo/panama-presentan-plan-maestrode-turismo-sostenible-post-covid-19

SENACYT (2018). La SENACYT organizó un taller de reflexión del estudio de prospectiva "Panamá 2040. Visión de un país exitoso." Recuperado de: https://www.senacyt.gob.pa/publicaciones/?p=23295

UNWTO. (2019). Panorama del turismo internacional, edición 2019. In Panorama del turismo internacional, edición 2019. Recuperado de: https://doi.org/10.18111/9789284421237

UNWTO. (2020). Las cifras de turistas internacionales podrían caer un $60-80 \%$ en 2020, informa la OMT. Recuperado de: https://www.unwto.org/es/news/covid-19-lascifras-de-turistas-internacionales-podrian-caer-un-60-80-en-2020\#: :text= Vargas Sánchez, A. (2020). Entender el turismo post-coronavirus: posibles escenarios. En Tpc19 1 Turismo Post COVID - 19. Recuperado de: https://aecit.org/uploads/public/DOCUMENTO.covid-19 y turismo.pdf 